\title{
Die Schmelzpunkte der Mineralien im Lichte neuerer Untersuchungen über das Gasthermometer.
}

\author{
Yon \\ Arteur L. Day und Ropert B. Sosman. ${ }^{1}$ \\ Mit 1 Tafel.
}

Als die Arbeiten des geophysikalischen Laboratoriums aufgenommen wurden, waren Temperaturen oberhalb $1150^{\circ}$ niemals genau mit dem Stickstoffthermometer gemessen worden. In der Reichs. anstalt war es üblich, die Ablesungen am Thermoelement oberhalb $1150^{\circ}$ so zu deuten, dals man zuerst die Kurre: Temperatur-Elektromotorische Kraft des Elementes zwischen 400 und $1150^{\circ}$ berechnete und diese dann extrapolierte. Die festen Temperaturen, die gewöhnlich auf Mefsvorrichtungen für hohe Temperaturen aufgedruckt werden, wie z. B. bei den direkt zeigenden Galvanometern von Siemens \& Halske, sind gewöhnlich aus dieser Extrapolation abgeleitet.

Die absolute Genauigkeit der Skala der Reichsanstalt ( 400 bis $1150^{\circ}$ ) schätzte man auf etwa $3^{\circ}$ bei $1150^{\circ}$, und der Fehler der Extrapolation oberhalb $1150^{\circ}$ mit dem Thermoelement ist sicherlich beim Schmelzpunkt des Platins mindestens zehnmal so grols. Es schien deshalb notwendig, eine neue Untersuchung über die hohen Temperaturen mit dem Gastbermometer zu unternehmen und insbesondere seinen Geltungsbereich eine beträchtliche Strecke über $1150^{\circ}$ auszudehnen, um eine feste Basis für die Mineraluntersuchungen in diesem Laboratorium zu erhalten.

Es wurde demnach i. J. 1904 eine derartige Arbeit begonnen, deren endgültige Resultate im vergangenen Jahr veröffentlicht worden sind. ${ }^{2}$ Es war nicht nur möglich, eine grölsere Genauigkeit zwischen 400 und $1150^{\circ} \mathrm{zu}$ erhalten, sondern auch die grund-

1 Aus dem Manuskript ins Deutsehe übertragen von I. Koplet-Berlin.

${ }^{2}$ Vorläufige Vitteilung: $\mathrm{D}_{\mathrm{Ay}}$ und Cemanen, Am. Journ. Sci. 26 (1908), 405. Endgültige Mitteilang: DAY und Sosmsx, Am. Juurn. Sci. 29 (1910), 93 bis 161. - R. B. Sosman, Am. Jomm. Sci. 30 (1910), 1-15.

Z. anore. Chem. Bd. ${ }^{2}$. 
legenden Messungen bis $1550^{\circ}$ auszudehnen, wobei eine Genauigkeit von ungefähr $2^{0}$ bei dieser Temperatur erreicht wurde.

Inzwischen waren die Temperaturmessungen mit Thermoelementen in diesem Laboratorium in der alten Weise bezeichnet worden durch Extrapolation der Kurve: Temperatur-Thermoelektrische Kraft. Die Zahlenergebnisse müssen daher durch die Differenz zwischen der alten und der neuen Temperaturskala korrigiert werden, wodurch es erforderlich wird, alle vorhandenen Temperaturangaben aus diesem Laboratorium auf die neue Skala umzurechnen. Diese Mitteilung enthält die aus dieser Neuberechnung sich ergebenden Werte.

\section{Schmelz- und Umwandlungspunkte.}

Der Schmelzpunkt einer reinen Substanz kann definiert werden als diejenige Temperatur, bei der Kristalle und Schmelze nebeneinander im Gleichgewicht bestehen können; ein „Umwandlungspunkt" als die Temperatur, bei der zwei verschiedene Kristallformen der Substanz nebeneinander im Gleichgewicht bestehen können. Nur die Zuführung oder die Entziehung von Wärme wird das Verschwinden der einen vor den beiden nebeneinander vorhandenen Formen bewirken. Sowohl Schmelzen wie Umwandlung werden demnach charakterisiert durch die gleichzeitig verlaufende Erscheinungen: Auftreten oder Verschwinden einer besonderen Kristallstruktur und Auftreten oder Verschwinden einer Wärmemenge.

\section{Schmelzintervalle.}

Die angegebene Definition bezieht sich auf reine Verbindungen; wenn das Material ein Gemisch oder eine feste Lösung ist, so hat es keinen Schmelzpunkt sondern ein Schmelzintervall mit theoretisch) bestimmten Temperaturgrenzen. Die sogenannten Schmelzintervalle von langsam schmelzenden Verbindungen ${ }^{1}$ (Quarz, Albit,

1 Tammann, Zeitschr. phys. Chem. 68 (1909), 257 und neuerdings Ditruer, 7. anorg. Ohem. 69 (1911), 273, haben verschiedene Schmelzpunktsbestimmungen aus diesem Laboratorium einer Kritik unterzogen, insbesondere die Messungen an Anorthit und Diopsid, die wegen ungenügender Kontrolle des Ofens und Überhitzung zu hoch sein sollten. Diese Kritik wird wahracheinlich rerursacht durch ein Mifsverständnis bei der Betrachtung 1. der Erscheinungen beim Schmelzen eines Silikats uud 2. der Lrgebnisse unserer Messungen. Wenn eine Substanz sich immer unterkühlt, bevor sie kristallisiert, so wird die Temperatur der Kristallisation mit der Geschwindigkeit der Abkühlung wechseln, d. h. sie wird eine 
Orthoklas) müssen sorgfältig unterschieden werder von den ,Schmelzintervallen" der Gemische (Kalk-Natriumfeldspate, unreine natür-

Zufallstemperatur sein, die von den Bedingungen des Versuches abhängt, und keine für die Substanz charakteristische physikalische Konstante darstellt. Wenn dieselbe Substanz so langsam schmilzt, dafs sie leicht beim Schmelzen überhitzt werden kann, so wird die Temperatur der vollständigen Verflüssigung mit der Erhitzungsgeschwindigkeit wechseln und sie ist dann gleichfalls eine Zufallstemperatur, die nur von den Bedingungen des Versuches abhängt. Reiner Quarz, Albit und Orthoklas sind solche Substanzen und ohne Zweifel kann man davon noch mehr finden. Die Temperatur-Zeitlurve ist keine zuverlässige Methode zur Bestimmung der Temperatur der Zustandsänderung eines solchen Stoffes.

Wenn andererscits die Substanz dieselbe kongtante Schmelatemperatur bei sehr verschiedenen Erhitzungsgeschwindigkeiten liefert, so ist die Schmelztemperatur eine physikalische Konstante, die für die Sub$\operatorname{stanz}$ charakteristiseh ist und nicht von den Bedingungen des Versuches abhängt. Diopsid und Anorthit sind solehe Stoffe, und die veröffentlichten Schmelzpunkte von reinem Anorthit und Diopsid, gegen die sich die Kritik riebtete, sind auf diese Weise ertalten. (Siehe Literaturnachweis 1, 4, 32, 44, 46.)

Wenn behauptet wird, wie dies neverdings Ditrler tut, dals das Thermoelement nicht die wahre Temperatur des Minerales zu irgend einer Zeit während des Erhitzens angibt, oder dafs die Energieänderung des Systemes nicht gleichzeitig mit dem Verschwinden der Kristallstruktur erfolgt, so ist ein experimenteller Beweis hierfür leicht mit dem "Abschreckungsofen" zu erhalten, dureh den sich die Frage ohne jeden Zweifel lösen lärst.

Nehmen wir an, dafs der Schmelzpunkt von Diopsid z. B. in der oben beschriebenen Weise bei $1391^{\circ}$ beobachtet wurde. Man bringt nun einen Teil ( $2 \mathrm{~g}$ ) der kristallisierten Beschickung in einen Abschreckungsofen, erhitzt ihn langsam auf $1388^{\circ}$ und hält die Temperatur bei diesem Punkt 1 Stunde lang konstant oder so lange, bis es nicht weiter fraglich sein kann, dals das Thermoelement und alle Teile der Beschickung dieselbe Temperatur besitzen. Iann entfernt mau den Boden des Ofens und läfst den Tiegel mit dem Diopsid plötzlich in ein Gefäls mit Quecksilber fallen, wodurch die Masse fast augenblicklich abgekühlt wird, ohne daf's Gelegenheit zu irgend einer weiteren Änderung gegeben ist. Findet man die Kristallstruktur des Diopsides bei der Prüfung unverändert, so liegt $1388^{\circ}$ unterhalb der Schmelztemperatur. Man bringt nun dasselbe Material noch einmal in den (ofen, erhitat auf $1396^{\circ}$, hält diese Temperatur hinreichend lange Zeit konstant und lälst die Beschickung wieder schnell wie vorher in das Quecksilber hinein fallen. Wenn der Diopsid jetzt als klares Glas erscheint, so müssen Zustandsänderung und alle dazu gehörigen Erscheinungen zwischen $1388^{\circ}$ und $1396^{\circ}$ erfolgt sein.

Um eir sichtbares Ergebnis von diesem besonderen Fall zu liefern, wurde dieser Versuch in unserem Laboratorium sowohl mit chemisch reinem Diopsid wie auch mit chemisch reinem Anorthit ausgeführt, und es wurden Photographien der Stoffe, wie sie nach Entfernung ans dem Ofen erschienen, aufgenommen, welche in den Figg. 1-4 (Tafel I) dargestellt sind. Diese Photo- 
liche Mineralien). Die ersteren schmelzen und werden amorph nur sehr langsam, aber ihr Schmelzintervall ist ein zeitliches, während das Schmelzintervall von Gemischen ein Temperaturintervall ist. Der reine Natriumfeldspat (Albit) schmilzt vollständig, wenn man ihn eine hinreichend lange Zeit etwas über seinen Schmelzpunkt erhitzt; wenn man dagegen ein Gemisch auf eine Temperatur innerhalb ihres Schmelzintervalles bringt, so tritt nur teilweise Schmelzung ein unter Ausbildung eines Gleichgewichtes, und es bleiben unbegrenzte Zeit kristallinische und flüssige Teile nebeneinander bestehen.

Die Erstarrungspunkte und -intervalle der Silikate sind abweichend von den Metallen - nicht so genau bestimmbar wie die Schmelzpunkte und -intervalle wegen der Neigung jener Stoffe zur Unterkühlung. Der Grad der Unterkühlung wechselt in weitem Umfang und wird von zahlreichen zufälligen Bedingungen beeinflufst. Die Cnterkühlung beläuft sich unter gewissen Bedingungen nur auf $1-2^{\circ}$, oder sie kann bei demselben Stuff unter anderen Verhältnissen so grofs werden, dals man das Material bei gewöhnlicher Temperatur als Glas erhält, das unbegrenzte Zeit in amorphem Zustand bleibt.

\section{Die wichtigsten Temperaturbestimmungen im geophysikalischen Laboratorium, 1905+1910.}

In Tabelle 1 sind die wichtigsten Fixtemperaturen, die in den letzten fünf Jahren in diesem Laboratorium bestimmt wurden, bezogen auf die neue Temperaturskala, zusammengestellt. Es sind dies keine neuen Bestimmungen. Die ursprünglichen mit dem Thermoelement oder auf optischem Wege erhaltenen Daten sind einfach auf die Skala von DAY und SosMax rom Jahre 1910 umgerechnet worden.

Alle thermoelektrischen Messungen in diesem Laboratorium wurden früher ausgewertet durch Berechnung einer Kurve der Form $e=a+b t+e t^{2}$, aus den Schmelzpunkten von Zink, Silber und Kupfer, und durch Benutzung dieser Kurve für die Extrapolation, wie seit der Veröffentlichung der Arbeit von HoLBons und $D_{A Y}$ i. J. 1899 allgemein gebräuchlich war. Gelegentlich wurde auch eine Funktion der Form $b=a+b e+c e^{2}$ benutzt.

graphien geben nebst den beigefügten Daten den absoluten Beweis dafür, dals der Sehmelzpunkt des Diopsids zwischen $1388^{\circ}$ und $1396^{\circ}$ liegt, und dafs der Schmelzpinkt des Anorthits zwischen $1547^{\circ}$ und $1561^{\circ}$ - nach der neuen Skala des Stickstoffthermometers - fällt. 
Die Kurve $e=f(b)$ gibt 'Temperaturwerte, die bei $1500^{\circ}$ etwa um $16^{\circ}$ zu niedrig sind, und die Kurve $t=f^{\prime}(e)$ gibt Werte, die bei $1500^{\circ}$ etwa um $33^{\circ} \mathrm{zu}$ niedrig sind. Bei $1100^{\circ}$ beträgt die Korrektur nur $1.5^{\circ}$, so dafs die Temperaturen bis $1100^{\circ}$ praktisch unverändert bleiben.

Die Angaben von Shephero und Raskin über die binären Systeme von Tonerde mit Kieselsäure. Kalk und Magnesia sind bereits umgerechnet in der deutschen Übersetzung der betreffenden Mitteilung. ${ }^{1}$ Die Neuberechnung der anderen Daten wird hier zum erstenmal mitgeteilt.

Die Schmelzpunkte, die die Basis für die jetzt geltende Skala bilden, sind folgende:

\begin{tabular}{|c|c|c|c|c|c|c|c|c|c|c|c|}
\hline Ziuk & • & & , & . & & & & & & & 418.2 \\
\hline Antimon & . & & . & . & . & . & . & & & & 629.2 \\
\hline Silber . & . & . & . & ' & . & . & & & & & 960.0 \\
\hline Gold. & . & . & . & . & . & . & & & & & 1062.4 \\
\hline Kupfer & . & & . & . & . & . & . & & & & 1082.6 \\
\hline Diopsid & . & . & 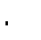 & . & . & . & & & & & 1391 \\
\hline Palladium & . & . & 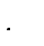 & . & . & . & & & & & 1549 \\
\hline Anorthit. & . & . & & 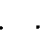 & . & . & & & & & 1550 \\
\hline Platinum & & " & & & 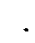 & . & & & & & 1755 \\
\hline
\end{tabular}

In der ersten Spalte von Tabelle 1 findet sich der Name des Minerals, in der zweiten seine Molekularformel, in der dritten die Umwandlung oder die Reaktion, die bei der in der vierten Spalte angegebenen Temperatur stattfindet; die beiden letzten Spalten enthalten den Namen des Beobachters und den Literaturnachweis. Die Nummern sind die der veröffentlichten Liste der Arbeiten aus diesem Laboratorium und die Zitate sind am Schlusse dieser Arbeit gegeben.

Eine Anzahl von Schmelz- und Umwandlungstemperaturen wurden nur angenähert bestimmt wegen der Trägheit oder der Langsamkeit der Änderung. Diese sind in Tabelle 2 zusammengestellt.

${ }^{1}$ Z. anorg. Chem. 68 (1910), 370-420. 
Tabelle 1.

Die wichtigsten Temperaturbestinmungen aus dem Geophysikalischen Laboratorium $1905-1910$.

\begin{tabular}{|c|c|c|c|c|c|}
\hline Substanz & Formel & Umwandlung & Temp. & Beobachter & $\begin{array}{l}\text { Literatur- } \\
\text { nachweis }\end{array}$ \\
\hline Quarz & $\mathrm{SiO}_{2}$ & $\begin{array}{c}\alpha-\text { in } \beta, \text { und } \\
\text { umgekehrt }\end{array}$ & 575 & $\begin{array}{l}\text { Wright und } \\
\text { Larsen }\end{array}$ & $\begin{array}{c}39,64 \\
(1909 / 10)\end{array}$ \\
\hline $\begin{array}{c}\text { Aluminiumsilikat, } \\
\text { (Sillimanit) }\end{array}$ & $\mathrm{Al}_{2} \mathrm{SiO}_{5}$ & Schmelzen & 1816 & $\begin{array}{c}\text { Shepherd u. } \\
\text { Rankin }\end{array}$ & $\begin{array}{c}\text { 41, 65 } \\
(1909 / 10)\end{array}$ \\
\hline $\begin{array}{l}\alpha \text {-Magnesiummeta- } \\
\text { silikat }\end{array}$ & $\mathrm{MgSiO}_{3}$ & $"$ & 1554 & $\begin{array}{c}\text { Allen, Wright } \\
\text { u. Clement }\end{array}$ & $13(1906)$ \\
\hline$"$ & $"$ & , & 1557 & $\begin{array}{l}\text { Allen und } \\
\text { White }\end{array}$ & $32(1909)$ \\
\hline $\begin{array}{c}\alpha \text {-Caleiummetasili- } \\
\text { kat,(Pseudowollasto- } \\
\text { nit) }\end{array}$ & $\mathrm{CaSiO}_{3}$ & $"$ & 1540 & $n$ & $6(1906)$ \\
\hline $\begin{array}{c}\alpha \text {-Calciumortho- } \\
\text { silikat }\end{array}$ & $\mathrm{Ca}_{2} \mathrm{SiO}_{4}$ & $"$ & 2130 & $\begin{array}{l}\text { Day und } \\
\text { Shepherd }\end{array}$ & $\underset{\substack{8,11 \\
(1906 / 7)}}{\text { u. } 19}$ \\
\hline $\begin{array}{c}\text { Eutektikum v. } \alpha \text {-Cal- } \\
\text { ciummetasilikat und } \\
\text { Cristobalit }\end{array}$ & $\mathrm{CaSiO}_{3} 77^{0 / 10}{ }^{1}$ & $"$ & 1426 & $"$ & $"$ \\
\hline $\begin{array}{l}\text { Eutektikum v. } \alpha \text {-Cal- } \\
\text { ciummetasilikat u. } \alpha \text { - } \\
\text { Calciumorthosilikat }\end{array}$ & $\begin{array}{l}\mathrm{CaSiO}_{8} 66 \% \\
\mathrm{Ca}_{2} \mathrm{SiO}_{4} 34 \%\end{array}$ & " & 1440 & $"$ & $"$ \\
\hline $\begin{array}{l}\text { Eutektikum ron } \\
\alpha \text {-Calciumorthosili. } \\
\text { kat und Kalk }\end{array}$ & $\begin{array}{l}\mathrm{Ca}_{2} \mathrm{SiO}_{4} 933_{0}^{\circ} \\
\mathrm{CaO} 7 \%\end{array}$ & $"$ & 2065 & $"$ & $"$ \\
\hline Tricalciumaluminat & 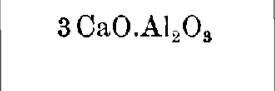 & $\begin{array}{l}\text { Dissoziation in } \\
\text { CaOu. Sehmelze }\end{array}$ & 1537 & $\begin{array}{l}\text { Shepherd u. } \\
\text { Rankin }\end{array}$ & $\begin{array}{c}\mathbf{4 1}, 65 \\
(1909: 10)\end{array}$ \\
\hline $\begin{array}{l}5: 3 \text { Verbindung v. } \\
\text { Kalk u. Tonerde }\end{array}$ & $5 \mathrm{CaO} .3 \mathrm{Al}_{2} \mathrm{O}_{3}$ & Schmelzen & 1382 & $"$ & $"$ \\
\hline Calciumaluminat & $\mathrm{CaO} \cdot \mathrm{Al}_{2} \mathrm{O}_{3}$ & $"$ & 1592 & , & : \\
\hline $\begin{array}{c}3: 5 \text { Verbindung } \nabla . \\
\text { Kalk u. Tonerde }\end{array}$ & $3 \mathrm{CaO} .5 \mathrm{Al}_{2} \mathrm{O}_{3}$ & $\begin{array}{l}\text { Dissoziation in } \\
\mathrm{Al}_{2} \mathrm{O}_{3} \text { und } \\
\text { Sehmelze }\end{array}$ & 1700 & $"$ & $"$ \\
\hline Eutektikum & $\begin{array}{rl}3 \mathrm{CaO} \cdot \mathrm{Al}_{2} \mathrm{O}_{3} & 9 \% \\
5 \mathrm{CaO} .3 \mathrm{Al}_{2} \mathrm{O}_{3} & 91 \%\end{array}$ & Schmelzen & 1378 & ", & ", \\
\hline
\end{tabular}

1 Die eutektischen Zusammensetzungen sind in Gewiebtsprozenten der angeführten Komponenten angegeben. 
Tabelle 1 (Fortsetzung).

\begin{tabular}{|c|c|c|c|c|c|}
\hline Substauz & Formel & Umwandlung & Temp. & Beobachter & $\begin{array}{c}\text { Literatur- } \\
\text { nachweis }\end{array}$ \\
\hline Eutektikum & $\begin{array}{l}5 \mathrm{CaO} .3 \mathrm{Al}_{2} \mathrm{O}_{3} 94 \% \\
\mathrm{CaO} \mathrm{Al}_{2} \mathrm{O}_{3} 6 \%\end{array}$ & Schmelzen & $1378^{\circ}$ & $\begin{array}{l}\text { Shepherd u. } \\
\text { Rankin }\end{array}$ & $\begin{array}{c}41,65 \\
(1909 / 10)\end{array}$ \\
\hline$"$ & $\begin{array}{c}\mathrm{CaO} \cdot \mathrm{Al}_{2} \mathrm{O}_{3} 73 \% \\
3 \mathrm{CaO} .5 \mathrm{Al}_{2} \mathrm{O}_{3} 27 \%\end{array}$ & $"$ & 1585 & $"$ & $"$ \\
\hline $\begin{array}{l}\text { Magnesium-Cal- } \\
\text { ciummetasilikat, } \\
\text { (Diopsid) }\end{array}$ & $\mathrm{MgSiO}_{3} \cdot \mathrm{CaSiO}_{3}$ & 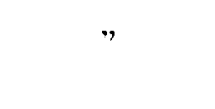 & $1391^{1}$ & $\begin{array}{l}\text { Day und } \\
\text { Sosman }\end{array}$ & $46(1910)$ \\
\hline$"$ & 1, & $\therefore$ & 1391 & $\begin{array}{l}\text { Allen und } \\
\text { White }\end{array}$ & $32(1906)$ \\
\hline $\begin{array}{l}\text { Eutektikum v. } 2 \text { fest. } \\
\text { Lösungen v. Diopsid } \\
\text { und } \alpha-\mathrm{MgSiO}_{3} \text { inein- } \\
\text { ander }\end{array}$ & $\begin{array}{c}95.5 \% \\
\text { Lösg. }\left\{\begin{array}{lll}62^{0} & \text { Dinps. } \\
38^{0} & \mathrm{MgSiO}_{3} \\
4.5 \% & 4^{0} & \mathrm{Diops}^{\circ} \\
\text { Lösg. } & 6^{0} & \mathrm{MgSiO}_{3}\end{array}\right.\end{array}$ &. & 1385 & $"$ & $32(1909)$ \\
\hline $\begin{array}{l}\text { Eutektikum v. Diop- } \\
\text { sid u. } \alpha-\mathrm{CaSiO}_{3}\end{array}$ & $\begin{array}{c}\mathrm{HgSiO} \cdot \mathrm{CaSiO} 60^{\circ} \\
\mathrm{CaSiO}_{9} 40^{\circ} \%\end{array}$ & $"$ & 1357 & ." & $"$ \\
\hline $\begin{array}{c}\text { Calcium-Alumi- } \\
\text { niumsilikat }(\Lambda \text { uorthit })\end{array}$ & $\begin{array}{l}\mathrm{CaAl} \mathrm{Si}_{2} \mathrm{O}_{8} \text { oder } \\
\mathrm{CaSiO}_{3} \cdot \mathrm{Al}_{2} \mathrm{SiO}_{5}\end{array}$ & " & 1552 & $\begin{array}{l}\text { Day und } \\
\text { Allen }\end{array}$ & $1,4(1905)$ \\
\hline$"$ & $"$ & , & $1550^{1}$ & $\begin{array}{l}\text { Day und } \\
\text { Sosman }\end{array}$ & $46(1910)$ \\
\hline Bytownit & Albit $_{1}$ Anorthit $_{5}$ & $"$ & $1516^{2}$ & $\begin{array}{l}\text { Day und } \\
\text { Allen }\end{array}$ & $\mathbf{1}, 4(1905)$ \\
\hline Labradorit & Albit $_{1}$ Anorthit 2 & $"$ & $1477^{2}$ & $"$ & " \\
\hline Andesin-Labradorit & Albit Anorthit $_{1}$ & $"$ & $1430^{2}$ & $"$ & .", \\
\hline Andesin & Albit $_{2}$ Anorthit & $"$ & $1375^{2}$ &. & $"$ \\
\hline Calciumkarbonat & $\mathrm{CaCO}_{3}$ & $\begin{array}{l}\text { Dissoziations- } \\
\text { druck = I Atm. }\end{array}$ & 898 & Johnston & $56(1910)$ \\
\hline Borax & $\mathrm{Na}_{2} \mathrm{~B}_{4} \mathrm{O}_{7}$ & Schmelzen & 741 & $\begin{array}{l}\text { Day und } \\
\text { Allen }\end{array}$ & $1,4(1905)$ \\
\hline Natriumchlorid & $\mathrm{NaCl}$ & $"$ & 800 & White & $44(1909)$ \\
\hline Natriumsulfat & $\mathrm{Na}_{2} \mathrm{SO}_{4}$ & $"$ & 884 & $"$ &, \\
\hline
\end{tabular}

1 Direkter Vergleich mit dem Stickstoffthermometer.

2 Als isomorphe Gemische von zwei Verbindungen haben diese Stoffe keineu Schmelzpunkt, sondern ein Schmelzintervall. Die Temperaturgrenzen dieses Intervalles sind wahrscheinlich eng und seine Existenz ist völlig verdeckt durch die geringe Gesehwindigkeit, womit diese Silikate das Gleichgewicht erreichen. Siehe die eingehende Besprechung in Mitteilung 1 (S. 60 bis 69 ) oder 4 (S. $40-50$ ). 
Tabelle 2.

Angenäherte Temperaturbestimmungen.

\begin{tabular}{|c|c|c|c|c|c|}
\hline Substanz & Formel & Umwandlung & $\begin{array}{l}\text { Temp. } \\
\text { in }\end{array}$ & $\begin{array}{l}\text { Beob- } \\
\text { achter }\end{array}$ & $\begin{array}{l}\text { Literatur- } \\
\text { nachweis }\end{array}$ \\
\hline $\begin{array}{c}\text { Cristobalit } \\
\text { (aus Quarz) }\end{array}$ & $\mathrm{SiO}_{2}$ & Schmelzen & ca. 1600 & $\begin{array}{l}\text { Day und } \\
\text { Shepherd }\end{array}$ & $\begin{array}{l}8,11,19 \\
(1906 / 7)\end{array}$ \\
\hline Quarz & $\mathrm{SiO}_{2}$ & $\begin{array}{l}\text { Umwandlung } \\
\text { in Cristobalit }\end{array}$ & über 800 & $\begin{array}{l}\text { Wright } \\
\text { u. Larsen }\end{array}$ & $\begin{array}{c}39,64 \\
(1909 / 10)\end{array}$ \\
\hline $\begin{array}{l}\text { Eutektikum }{ }^{1} \text { y. Cri- } \\
\text { stobalit u. Sillimanit }\end{array}$ & $\begin{array}{c}\mathrm{SiO}_{3}, 80 \% \\
\mathrm{Al}_{2} \mathrm{SiO}_{5}, 20 \%\end{array}$ & Sehmelzen & $\begin{array}{c}\text { Etwas unter } \\
1600\end{array}$ & $\begin{array}{l}\text { Shepherd } \\
\text { u. Rankin }\end{array}$ & $\begin{array}{c}41,65 \\
(1909 / 10)\end{array}$ \\
\hline $\begin{array}{l}\text { Eutektikum v. Co- } \\
\text { rund u. Sillimanit }\end{array}$ & $\begin{array}{rl}\mathrm{Al}_{2} \mathrm{O}_{3}, 3 \% & 3 \% \\
\mathrm{Al}_{2} \mathrm{SiO}_{5}, 97 \%\end{array}$ & $"$ & ca. 1810 & , & $"$ \\
\hline $\begin{array}{l}\text { Magnesiummeta- } \\
\text { silikat }\end{array}$ & $\mathrm{MgSiO}_{3}$ & $\begin{array}{c}\text { Umwandlg. } \alpha \text {-in } \beta \text { - } \\
\text { (Clinoenstatit), und } \\
\text { umgekehrt }\end{array}$ & ca. 1375 & $\begin{array}{l}\text { Allen u. } \\
\text { White }\end{array}$ & $82(1909)$ \\
\hline $\begin{array}{l}\beta \text {-Caleiummetasili- } \\
\text { kat, (Wollastonit) }\end{array}$ & $\mathrm{CaSiO}_{3}$ & $\begin{array}{l}\text { Umwandlung in } \\
\alpha \text {-CaSiO (Pseudo- } \\
\text { wollastonit) und } \\
\text { umgekehrt }\end{array}$ & 1180 & " & $\begin{array}{c}6,32 \\
(1906 / 7)\end{array}$ \\
\hline Calciumorthosilikat & $\mathrm{Ca}_{2} \mathrm{SiO}_{4}$ & $\begin{array}{l}\text { Umwandlg. } \text {. } \alpha \text { in } \\
\beta \text { und umgekehrt }\end{array}$ & 1420 & $\begin{array}{l}\text { Day und } \\
\text { Shepherd }\end{array}$ & $\begin{array}{l}8,11,19 \\
(1906,9)\end{array}$ \\
\hline$"$ & $"$ & $\begin{array}{l}\text { Umwandlg. } v \cdot \beta \text { in } \\
\gamma \text { und amgekehrt }\end{array}$ & 675 & $"$ & $"$ \\
\hline Tricalciumsilikat & $3 \mathrm{CaO}_{\mathrm{SiO}}$ & $\begin{array}{l}\text { Bildg. v. } 2 \text { Phasen } \\
\text { durch Dissoziation }\end{array}$ & ca. 1900 & $\begin{array}{l}\text { Shepherd } \\
\text { u. Rankin }\end{array}$ & 61 (1911) \\
\hline $\begin{array}{l}\text { Eutektikum von } \\
\text { Spinell u. Periklas }\end{array}$ & $\begin{array}{l}\mathrm{MgO} \cdot \mathrm{Al}_{2} \mathrm{O}_{3} \\
\text { und } \mathrm{MgO}^{2}\end{array}$ & Schmelzen & ca. 1950 & " & $\begin{array}{c}41,65 \\
(1909 ; 10)\end{array}$ \\
\hline Albit & $\mathrm{NaAlSi}_{3} \mathrm{O}_{8}$ & $"$ & unter $1200^{4}$ & $\begin{array}{l}\text { Day u. } \\
\text { Allen }\end{array}$ & $\begin{array}{c}1,4 \\
(1905)\end{array}$ \\
\hline Oligoklas-Andesin & Albit $_{3}$ Anorthit & " & $1345^{2}$ & , &, \\
\hline Mikroklin & $\mathrm{KAlSi}_{3} \mathrm{O}_{3}$ &, & unter $1200^{4}$ &, & , \\
\hline Andalusit & $\mathrm{Al}_{2} \mathrm{SiO}_{5}$ & $\begin{array}{l}\text { Umwandlung in } \\
\text { Sillimanit }\end{array}$ & $\begin{array}{c}1300 \\
\text { und höher }\end{array}$ & $\begin{array}{l}\text { Shepherd } \\
\text { u. Rankin }\end{array}$ & $\begin{array}{c}41,65 \\
(1909 ; 10)\end{array}$ \\
\hline Cyanit od. Disthen & " & $\because:$ & $\begin{array}{c}1300 \\
\text { und höher }\end{array}$ & $n$ & " \\
\hline $\begin{array}{l}\alpha^{\prime} \text {-Magnesiummeta- } \\
\text { silikat, (Enstatit) }\end{array}$ & $\mathrm{MgSiO}_{3}$ & $\begin{array}{l}\text { Umwandlg. aus d. } \\
\text { unstab. Form in } \beta \\
\mathrm{MgSiO}_{3} \text { (Klino- } \\
\text { enstatit) }\end{array}$ & $1300^{3}$ & $\begin{array}{c}\text { Allen, } \\
\text { Wright u. } \\
\text { Clement }\end{array}$ & $13(1906)$ \\
\hline $\begin{array}{c}\beta^{\prime} \text {-Magnesiummeta- } \\
\text { silikat, monokliner } \\
\text { Amphibol }\end{array}$ & $"$ & $"$ & $\begin{array}{c}1150 \\
\text { und höher }\end{array}$ & $"$ & $"$ \\
\hline $\begin{array}{c}\gamma^{\prime} \text {-Magnesiummeta- } \\
\text { silikat, rbombischer } \\
\text { Amphibol } \overline{5}\end{array}$ & $"$ & $"$ & $\begin{array}{c}1150 \\
\text { und höher }\end{array}$ & , & : \\
\hline
\end{tabular}


Anmerkungen zu 'Tabelle 2:

1 Die eutektischen Zusammensetzungen sind in Gewichtsprozenten der angefübrten Verbindungen angegeben.

${ }^{2}$ Siehe unter ${ }^{2}$ S. 7 (Tab. 1).

${ }^{3}$ Diese Temperaturen unterscheiden sich von den anderen der Tabelle dadurch, dais sie nicht feste physikalisehe Punkte sind. Die Änderung erfolgt aus einer instabilen in eine stabile Form und ist nicht umkehrbar. Die Zahlen bezeichnen nur die Temperaturen, bei der die Umwandlung schnell genug verläuft, um in verbältnismälsig kurzer Zeit beobachtet werden zu können.

${ }^{4}$ Der reinste natürliche Albit zeigte Spuren von Schmelzen, wenn er einige Minuten auf $1200^{\circ}$ erhitzt wurde und auch bei vierstündigem Erhitzen auf $1100^{\circ}$. Kleine Mengen gewisser Verunreinigungen können jedoch den Sehmelzpunkt beträchtlich erniedrigen, während andere ihn erhöhen. Schmelzpunktsbestimmungen an chemisch reinem Albit oder Orthoklas sind nicht ausgeführt worden. Man kann deswegen höchstens sagen, dafs der Schmelzpunkt wahrseheinlich unter $1200^{\circ}$ liegt. Beim Mikroklin liegen die Verhältnisse ähnlich.

${ }^{5}$ Diese beiden Mineralien sind von verschiedenen Autoren Kupfferit genannt worden.

\section{Literaturnachweis.}

1. Isomorphismus und thermische Eigensehaften der Feldspate. I. Thermische Unters. v. Arther L. Dar und E. T. Alien. II. Optische Unters. v. J. P. Indings, mit einer Einleitung von Geonge F. Becker. Publikation Nr. 31, Carnegie Institution of Washington (1905).

4. Der Isomorphismus und die thermischen Eigenschaften der Feldspate. Arthur L. Day und E. T. Alles, Zeitschr. phys. Chem. 54 (1905), 1-54.

6. Wollastonit und Pseudo-Wollastonit, polymorphische Formen des Calciummetasilikats. E. T. ALIEN und W. P. White, Mit optischen Unters, v. Fred. Eugene Wright, Am. Journ. Sci. [4] 21 (1906), 89-108.

7. Quarzglas. Artuur I. Dax und E. S. Suepuend Science, N. S. 23, No. 591 (1906), p. $670-672$.

8. Die Kalk-Kieselsäurereihe der Mineralien. Arthur L. Day und E. S. Shrpaerd, Journ. Amer. Chem. Soe. 28 (1906), 1089-1114.

11. Die Kalk-Kieselsäurereihe der Mineralien. Artaur L. DAY und E. S. Shepherd, m. opt. Unters, v. Fred. Eugene Wright, Amer. Journ. Sci. [4] 22 (1906), $265--302$.

13. Mineralien der Zusammensetzung $\mathrm{MgSiO}_{3}$. Ein Fall von Tetramor phismus. E. T. Allen, Fred. Eugene Wright und J. K. Clement, Amer. Journ Sci. [4] 22 (1906), 385-438.

19. Die Kalk-Kieselreihe der Mineralien. Arthor L. Day, E. T. Allen, E. S. Shepherd, W. P. White und Fred. Eugese Wright, Tscherm. Min. petr. Mitt. 26 (1907), $169 \rightarrow 232$.

32. Diopsid und seine Beziehungen zu Calcium- und Magnesiummetasilikat. E. T. Allen und W. P. White; m. opt. Unters. v. Fred. Eugene Wriant und Esper S. Larsen. Amer. Journ. Sei. [4] 27 (1909), 1-47.

39. Quarz als geologisches Thermometer. Fred. Eugene Wricat und Esper S. Laksen, Amer. Journ. Sci. [4] 27 (1909), 421-447. 
41. Die binären Systeme von Tonerde $\mathrm{m}$. Kieselsäure, Kalk und Magnesia. E. S. Shepherd und G. A. Rankin; m. opt. Unters. v. Fred. Eugene Wnght, Amer. Journ. Sei. [4] 28 (1909), 293-333.

44. Schmelzpunktsbestimmungen. WaLter P. White, Aner. Journ. Sci. [4] 28 (1909), 453-473. Sehmelzpunktsmethoden bei hoben Temperaturen. Walter P. White, Amer Journ. Sei. [4] 28 (1909), 474-489. Z. anorg. Chem. 69 (1911) 305-352.

46. Das Stickstoffthermometer von Zink bis Palladium. Arthor L. Day und Robert B. Sosman; m. einer Unters. der Metalle v. Eugene T. Allen, Amer. Journ. Sei. [4] 29 (1910), 93-161.

54. Das Platinum-Rhodium Thermoelement von $0-1755^{\circ}$. Roвenr B. Sosman, Amer. Joum. Sci. [4] $30(1910), 1-15$.

56. Die thermische Dissoziation von Calciumkarbonat. Jонм Jонsтом, Journ. Amer. Chem. Soe. 32 (1910), 93ิ8-946.

61. Vorläufiger Bericht über das ternäre System $\mathrm{CaO}-\mathrm{Al}_{3} \mathrm{O}_{3}-\mathrm{SiO}_{2}$ : Unters. über die Konstitution der Portlandzement-Klinker. E. S. Suerefrod und G. A. Rankin; nebst opt. Untersuchungen von Fred. Eugene $W_{\text {Riaut, Jour. }}$ Ind. and Enq. Chem. $\mathbf{3}$ (1911), 211-227. Z. anorg. Chem. 71 (1911), 19-64.

64. Quarz als geologisches Thermometer. F. E. Whigut und E. S. LARSEN, Z. anorg. Chem. 68 (1910), 338-369.

65. Die binären Systeme von Tonerde mit Kieselsäure, Kalk und Magnesia. E. S. Shepherd und G. A. RANkin. Nebst optischen Untersuchungen von F. E. Wright, Z. anorg. Chem. $68(1910)$, 370-420.

\section{Tafelerklärung.}

Fig. 1. Chemisch reiner Diopsid, Element $R, 14100$ Mikrovolt $=1388^{\circ}$; Zeit: 1 Stunde; gekreuzte Nicols; 250 malige Vergrölserung. Kristallinisch. Die Interferenzfarben zeigeu sich in Banden urn die Kanten der Kristallfragmente.

Fig. 2. Chemisch reiner Diopsid, Element $R, 14200$ Mikrovolt $=1396^{\circ}$; Zeit: 1/4 Stunde; photographiert mit gekreuzten Nicols; Vergrörserung 40 fach linear. Glas.

Fig. 3. Chemisch reiner Anorthit, Element $R, 15950$ Mikrovolt $=1547^{\circ}$; Zeit: $1 / 4$ Stunde; gekreuzte Nicols; Vergrölserung 250 fach linear; kristallinisch, deutliche Zwillingsbildung.

Fig. 4. Chemisch reiner Anorthit, Element $R, 16100$ Mikrovolt $=1561^{\circ}$; Zeit: $1 / 4$ Stunde; gekreuzte Nicols; 125 fache Vergölserung, Glas.

Washington, Geophysical Laboratory, Carnegie Institution, Mär: 1911.

Bei der Redaktion eingegangen am 8. April 1911. 